\title{
Pre-trained based CNN model to identify finger vein
}

\author{
Subha Fairuz ${ }^{1}$, Mohamed Hadi Habaebi ${ }^{2}$, Elsheikh Mohamed Ahmed Elsheikh ${ }^{3}$ \\ ${ }^{1,2}$ Department of Electrical and Computer Engineering, International Islamic University Malaysia, Malaysia \\ ${ }^{3}$ Universiti Kuala Lumpur British Malaysian Institute, Selangor, Malaysia
}

\begin{tabular}{l}
\hline \hline Article Info \\
\hline Article history: \\
Received Mar 5, 2019 \\
Revised May 8, 2019 \\
Accepted May 24, 2019 \\
\hline
\end{tabular}

Keywords:

Alexnet

Biometric identification

Convolutional Neural Network

(CNN)

Finger-vein recognition

Transfer learning

\begin{abstract}
In current biometric security systems using images for security authentication, finger vein-based systems are getting special attention in particular attributable to the facts such as insurance of data confidentiality and higher accuracy. Previous studies were mostly based on finger-print, palm vein etc. however, due to being more secure than fingerprint system and due to the fact that each person's finger vein is different from others finger vein are impossible to use to do forgery as veins reside under the skin. The system that we worked on functions by recognizing vein patterns from images of fingers which are captured using near Infrared (NIR) technology. Due to the lack of an available database, we created and used our own dataset which was pre-trained using transfer learning of AlexNet model and verification is done by applying correct as well as incorrect test images. The result of deep convolutional neural network (CNN) based several experimental results are shown with training accuracy, training loss, Receiver Operating Characteristic (ROC) Curve and Area Under the Curve (AUC).
\end{abstract}

Copyright $@ 2019$ Institute of Advanced Engineering and Science. All rights reserved.

\section{Corresponding Author:}

Mohamed Hadi Habaebi,

Department of Electrical and Computer Engineering,

Faculty of Engineering, International Islamic University Malaysia,

Jalan Gombak, 53100 Kuala Lumpur, Malaysia.

Email: habaebi@iium.edu.my

\section{INTRODUCTION}

Biometric identification-based technology has seen an outstanding growth in the recent years and among them finger vein-based identification is mention worthy due to its efficiency in providing security and accuracy [1]. The security is ensured in this system from forgery and interference due to the fact that the vein network is located internally within the body and cannot be affected by outside disturbances such as dirtiness, humidity [2,3]. Finger vein-based systems have benefits in comparison to other conventional techniques since it is non-intrusive and device size is smaller. Thus, it possesses great future prospects in the biometric technology field [4].

Locating veins under the skin means revealing venous networks using sophisticated camera technologies in order to make these networks distinguishable and a technology which can accomplish this feat is known as line tracking technique [5]. Among other mention-worthy techniques such as Far-infrared (FIR) and Near-infrared (NIR) is able to detect finger veins accurately. NIR and FIR both camera technologies possess the capability of infiltrating $5 \mathrm{~mm}$ under the skin tissue and illuminating 740-940 nanometers [6] whereas human eyes are only able to see in between 380-720 nanometers in the visible light spectrum [7]. FIR detects tissues with higher temperature adjacent to the outside skin while NIR captures venous networks which carries blood due to the blood having the ability to absorb infrared radiation. [8].

Though the advances in the finger-vein based biometric technology are terrific but still processes for vein extraction from fingers remains dependent on four methods which are local-invariant based methods [9, 10], statistical based methods [11], sub-space learning-based techniques [12] and vessel extraction $[13,14]$. It is possible to extract venous networks as a line like structures [15], as minutiae [16], as curvature 
$[7,8]$ or as a network of dark lines as apart of vessel extraction. Besides these techniques, there are other ways of recognition such as tracking times and curvature values, thresholds and neighborhood number. [17]

In a traditional finger vein authentication system, there are four steps: Image Acquisition, Pre-processing, feature extraction and matching or verification. To ensure higher accuracy rate of finger vein recognition process training-base methods are more efficient than a non-training-based algorithm. Convolutional neural network $(\mathrm{CNN})$ based network model contains an input layer, several hidden layers, output layer [18, 19]. Generally, a CNN network contains an input layer, several hidden layers, output layer. An architecture of Alexnet is illustrated in Figure 1, where the first layer is the input image $224 \times 224$ then several hidden layers and finally the output layer.

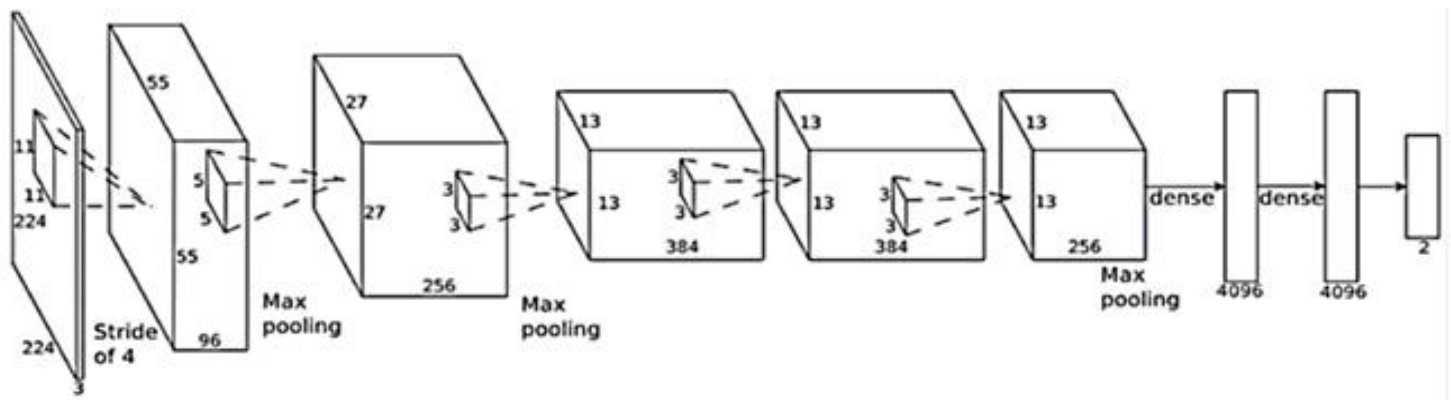

Figure 1. Architecture of Alexnet [20]

Common hidden layers are convolution layer, Rectified Linear Units (ReLu), pooling, normalization etc. Hundreds of images can be modified by this CNN models, for this reason to train a network GPU is needed otherwise difficult to work with complex models. [21] However, more difficult security problem such as, spoofing attacks in finger vein database [22] also getting impressive result. Table 1 shows comparison of related works [23].

Identification accuracy achieved in the research [17] for databases such as HKPU, FV-USM, SDUMLA is $98 \%$ while for database UTFVP the rate is $99.4 \%$. A vision-based device was proposed and developed in [8] which is a promising device for phlebotomy procedures. A deep learning-based approach known as LeNet-5 model of CNN which used convolutional neural network for finger vein identification in [24] showcasing a recognition rate of $96 \%$ in the Windows-based system and $100 \%$ in Linux based system. AlexNet of CNN approach was used in [25] for finger vein recognition which produced an accuracy rate of 99.53\%. VGG Net-16 of CNN was used for developing a finger vein identification system in [26] which showcased a recognition rate of $97.7 \%$.

Table 1. Comparison of related works [23]

\begin{tabular}{|c|c|c|c|c|}
\hline Author- year & Topic & Method & Strength & Limitation \\
\hline $\begin{array}{l}\text { Rig Das, Emanuele } \\
\text { Maiorana, Patrizio } \\
\text { Campisi, 2018 [17] }\end{array}$ & $\begin{array}{l}\text { Convolutional Neural } \\
\text { Network for Finger-Vein- } \\
\text { based Biometric } \\
\text { Identification }\end{array}$ & $\begin{array}{l}\text { Applied deep learning } \\
\text { method using } \\
\text { existence public } \\
\text { databases }\end{array}$ & $\begin{array}{l}\text { Used four public } \\
\text { databases and establish } \\
\text { CNN based finger vein } \\
\text { identification system. }\end{array}$ & $\begin{array}{l}\text { Static images used only, } \\
\text { didn't created own } \\
\text { database for the } \\
\text { experiments }\end{array}$ \\
\hline $\begin{array}{l}\text { Kazi Istiaque Ahmed, } \\
\text { Mohamed Hadi Habaebi, } \\
\text { Md Rafiqul Islam and } \\
\text { Nur Aishah Bt } \\
\text { Zainal,2017 [8] }\end{array}$ & $\begin{array}{l}\text { Enhanced Vision Based } \\
\text { Vein Detection System }\end{array}$ & $\begin{array}{l}\text { NIR imaging } \\
\text { Technique and } \\
\text { contrast limited } \\
\text { adaptive equalization } \\
\text { to process images }\end{array}$ & $\begin{array}{l}\text { Image quality is better } \\
\text { and detecting the vein } \\
\text { in real time }\end{array}$ & $\begin{array}{l}\text { Expensive hardware is } \\
\text { used to capture images }\end{array}$ \\
\hline $\begin{array}{l}\text { K. S. Itqan, A. R. } \\
\text { Syafeeza, F. G. Gong, N. } \\
\text { Mustafa, } \\
2016[24]\end{array}$ & $\begin{array}{l}\text { User Identification } \\
\text { System based on finger- } \\
\text { vein Patterns using } \\
\text { convolutional neural } \\
\text { network }\end{array}$ & $\begin{array}{l}\text { LeNet-5 network } \\
\text { trained finger vein } \\
\text { images }\end{array}$ & $\begin{array}{l}\text { Own databased is used } \\
\text { and experimental } \\
\text { result is pretty } \\
\text { acceptable }\end{array}$ & $\begin{array}{l}\text { Image quality is low, } \\
\text { recognition is not in real } \\
\text { time. }\end{array}$ \\
\hline $\begin{array}{l}\text { Wenjie Liu, Weijun Li, } \\
\text { Linjun Sun, } 2017 \text { [25] }\end{array}$ & $\begin{array}{l}\text { Finger Vein Recognition } \\
\text { Based on Deep Learning }\end{array}$ & $\begin{array}{l}\text { AlexNet based deep } \\
\text { learning method used } \\
\text { for finger vein } \\
\text { recognition }\end{array}$ & $\begin{array}{l}\text { Result beats } \\
\text { expectancy and } \\
\text { showed CNN is better } \\
\text { than traditional } \\
\text { algorithms }\end{array}$ & $\begin{array}{l}\text { Image quality is not } \\
\text { pleasing, can be more } \\
\text { accurate }\end{array}$ \\
\hline $\begin{array}{l}\text { Hyung Gil Hong, Min } \\
\text { Beom Lee and Kang } \\
\text { Ryoung Park, } 2017 \text { [26] }\end{array}$ & $\begin{array}{l}\text { Convolutional Neural } \\
\text { Network-Based Finger- } \\
\text { Vein Recognition Using } \\
\text { NIR Image Sensors }\end{array}$ & $\begin{array}{l}\text { Two public databases } \\
\text { are used to prove } \\
\text { CNN }\end{array}$ & $\begin{array}{l}\text { Non-trained classes } \\
\text { can be recognized and } \\
\text { reduce complexity }\end{array}$ & $\begin{array}{l}\text { Proposed method is quite } \\
\text { more complex than } \\
\text { existing methods. }\end{array}$ \\
\hline
\end{tabular}


In this research, we are using human finger vein data to acknowledge human identity using our finger vein collector device. However, we assembled more than one copies of a finger and keep as one information which later accommodated for training. In the next section, we illustrated our finger vein recognition approach. In the third section, we explained our experimental results and analysis, and also the last section is about our future vision of this work and conclusion.

\section{RESEARCH METHOD}

The technology which is revolutionizing the research field of computer vision is $\mathrm{CNN}$ and it is continuing to increase the advancement of accurate image classification besides playing a key role in performing feature extraction and recognition tasks such as image retrieval, object detection, semantic segmentation [27]. Due to the fact that the modern era is technologically advanced meaning enhanced computing power and GPU acceleration which makes it possible for the robust and efficient use of CNN. Based on deep CNN a model known as AlexNet [28] which consists of two fully-connected layers, five convolutional layers, and a softmax output layer. This network is coherent, unlike other simplistic networks which include Rectified Linear Units (ReLU), dropout and overlap pooling [29].

\subsection{Developed a device and construct the database}

To create an information and capture finger vein images, we create a tool which can take finger vein pictures by exploitation transmission imaging. There is a space to insert the finger and taking finger vein images, however, the light source illuminates from the underside of the tool as well as images captured from the top. To induce high-quality information, we covered the surroundings, used CMOS camera, take away background noises, NIR light sources, detected the region of interest (ROI) and stored the images in two different folders, one for training and another one for testing.

We collected twice total 12 finger's data and save those according to person's identity for both training and testing purpose. For training, we stored 100 images whereas, 30 images for testing purpose, each folders image are altered furthermore and removed the noises, detected the ROI and kept the data with the support of MATLAB [30].

\subsection{Training of CNN model}

Alexnet can be trained from the initial stage of the network by using random adjustment of weights or it can be trained a pre-train model using transfer learning features, which is faster and also a small number of images needs to train the model. There are five convolutional layers and three fully-connected (FC) layer, in each convolutional layer consist of convolution, Rectified Linear Unit (ReLU) pooling feature detection layers and fully-connected layers connect every neuron of a layer to another layer and provide classification output using softmax function [29].

a. To proceed with the transfer learning process, we pre-processed our input image from $747 \times 366 \times 3$ to $227 \times 227 \times 3$

b. First convolutional layer's (conv1) filter size is $11 \times 11 \times 3$ where stride and padding are 4,0 respectively.

c. ReLu confirms a negative value to zero and keeps it positive

d. Max pooling reduces the number of parameters by using non-linear downsampling and normalization kernel size is $3 \times 3$. When an input image is finished processing then we get an image with the dimension $55 \times 55 \times 96$

e. A similar technique is used for other convolutional layers where conv2, conv3, conv4, conv5 generates the following dimensions $27 \times 27 \times 256,13 \times 13 \times 384,13 \times 13 \times 384,13 \times 13 \times 256$.

f. In conv 2 filter size is $5 \times 5$ with stride 1 . In the similar manner conv3, conv4, conv 5 uses $3 \times 3$ filter and stride 1 . After getting fully-connected layers, we dropout $50 \%$.

\section{RESULTS AND ANALYSIS}

A simple confusion matrix is a table of four types of combinations of actual and predicted value which can measure the performance of a classifier on a test dataset based on that actual values showed in the following Table 2. If the predicted result is the positive and actual result is also positive then it is known as the true positive (TP). Again, If the predicted result is negative but the actual result is positive then it is known as the true negative (TN). Contrarily, If the predicted result is the positive but actual result is negative then that term known as false positive (FP). Furthermore, If the predicted result is the negative and actual result is also negative then it is known as the false negative (FN). 
Table 2. Confusion matrix

\begin{tabular}{lcc}
\hline & Confusion Matrix \\
\hline & Predicted Positive (P) & Predicted Negative (N) \\
Actual Positive (P) & True Positive (TP) & False Negative (FN) \\
Actual Negative (N) & False Positive (FP) & True Negative (TN) \\
\hline True positive rate (TPR) or, Sensitivity, TPR=TP/P=TP/(TP+FN); \\
True Negative rate (TNR) or, Specificity, TNR=TN/N=TN/(TN+FP); \\
False Positive rate (FPR), FPR=1-TNR=FP/(TN+FP), So, Accuracy \\
$($ ACC) $=(\mathrm{TP}+\mathrm{TN}) /(\mathrm{TP}+\mathrm{FP}+\mathrm{FN}+\mathrm{TN})$
\end{tabular}

True positive rate or sensitivity against False positive rate is known as receiver operating characteristic (ROC) curve which is the curve of probability and it shows the performance of a classification model. Area Under the curve (AUC) indicates the quality of the ROC curve. AUC will be 1 when the ROC curve has an ideal classifier however AUC value zero indicates that the experiment is predicting the false classes $[23,30,31]$.

\subsection{Actual training data and test data}

\subsubsection{Experiment 1}

Using transfer learning on pre-trained Alexnet, we experimented through various type of testing to get a feasible result for our finger vein identification system.

For the first experiment, we used 50 images from each of the four classes to train our network where the highest number of the epoch was 8 and the mini-batch size was 20 shown in Figure 2(a) which took a total time of $37.5 \mathrm{~s}$ for the test to run while the predictive ability is $91 \%$ and AUC is 0.942 . As we can see in the first experiment performance is really pleasant. A better model means lower the losses, the loss value indicates how strong or poorly a specific model is performing after each iteration, However, one would assume that the reduction of loss after each, or several, iteration(s). From Figure 2(b), we can see that training loss going downward from around 1.4 to 1 .

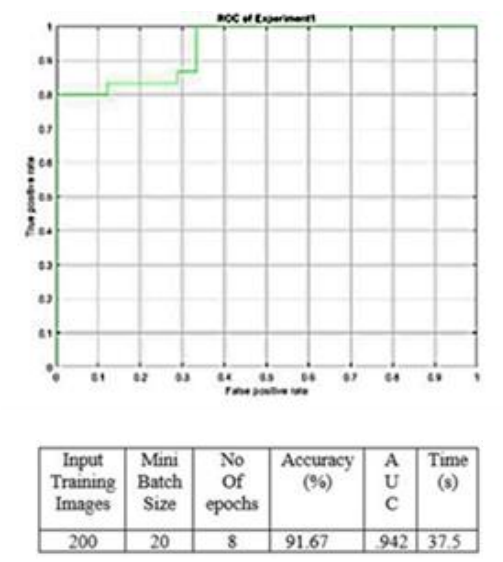

(a)

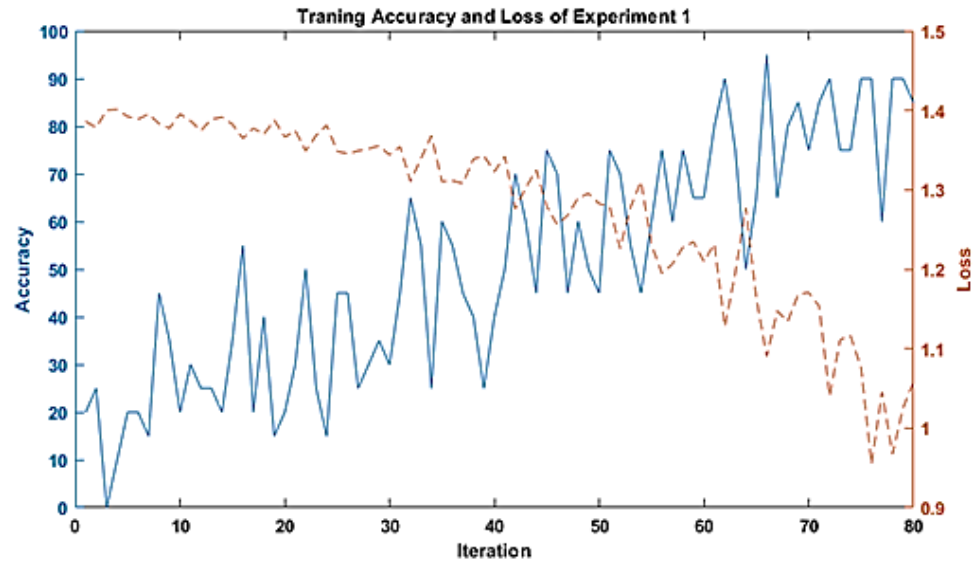

(b)

Figure 2. (a)ROC curve of Experiment 1, (b) Training accuracy (\%) and loss of Experiment 1

\subsubsection{Experiment 2}

On the other hand, for the second experiment we used 50 images per finger to train the network which took an execution time of 178s which is higher than the previous experiment. Although the second test took a slightly higher time for processing the data, the predictive efficiency is $100 \%$ and AUC is 1 which is an excellent in terms of performance. It is also notable that, the number of epochs is 90 and minibatch size was 32 according to Figure 3(a), which is better in comparison to the first experiment still, this experiment delivered a better result. In this experiment from Figure 3(a), we can see that AUC is in ideal situation and predictability is $100 \%$ while Figure 3(b) showcases training loss drop to 0. 


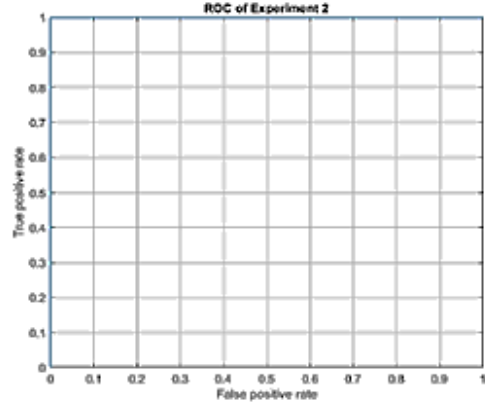

\begin{tabular}{|c|c|c|c|c|c|}
\hline $\begin{array}{c}\text { Input } \\
\text { Training } \\
\text { Images }\end{array}$ & $\begin{array}{c}\text { Mini } \\
\text { Batch } \\
\text { Size }\end{array}$ & $\begin{array}{c}\text { No } \\
\text { Of } \\
\text { epochs }\end{array}$ & $\begin{array}{c}\text { Accuracy } \\
(\%)\end{array}$ & $\begin{array}{c}\text { U } \\
\text { U }\end{array}$ & $\begin{array}{c}\text { Times } \\
\text { (s) }\end{array}$ \\
\hline 200 & 32 & 90 & 100 & 1 & 278 \\
\hline
\end{tabular}

(a)

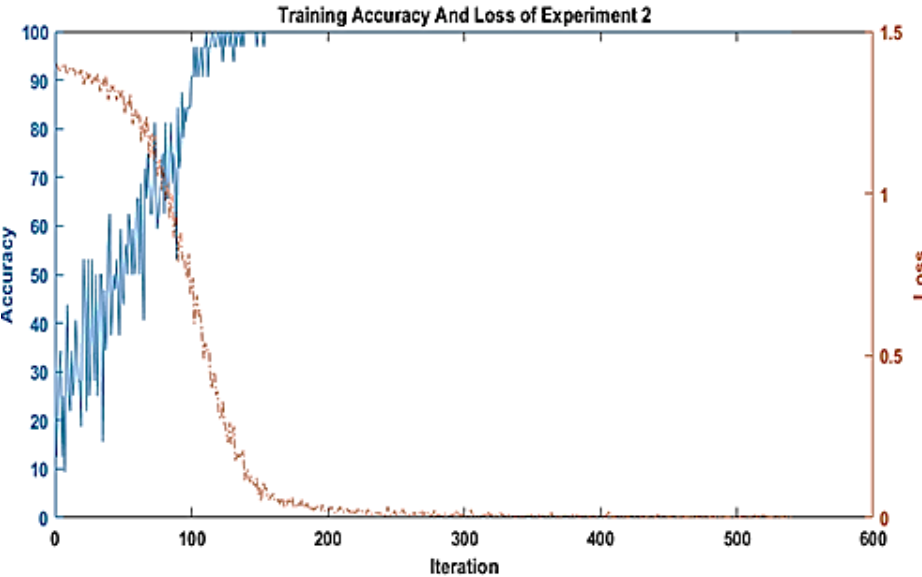

(b)

Figure 3. (a) ROC curve of experiment 2, (b) Training accuracy (\%) and loss of experiment 2

\subsubsection{Experiment 3}

Lastly, in the third experiment, we trained the network with a minimal number of images which was 80 in total, taking 20 images from each class. Furthermore, epoch count was 20 and mini-batch size was 16 in Figure 4(a) and the total predictive ability is $100 \%$ which is better than the first and second experiment because this experiment, number of epoch and minibatch size is less than experiment 1 and 2 . AUC is high which is 1 and took less time than previous experiments and accuracy 100\% according to Figure 4(b) where loss value dropped to $\sim 0$. Thus, if we do comparison between these three experiments, third experiment's results are better than first and second experiments.

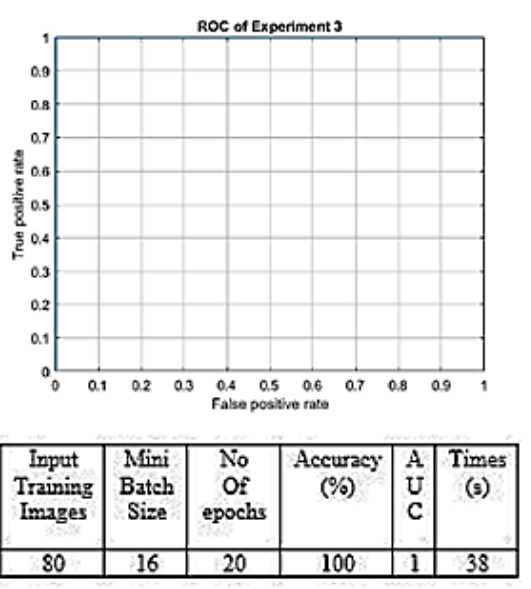

(a)

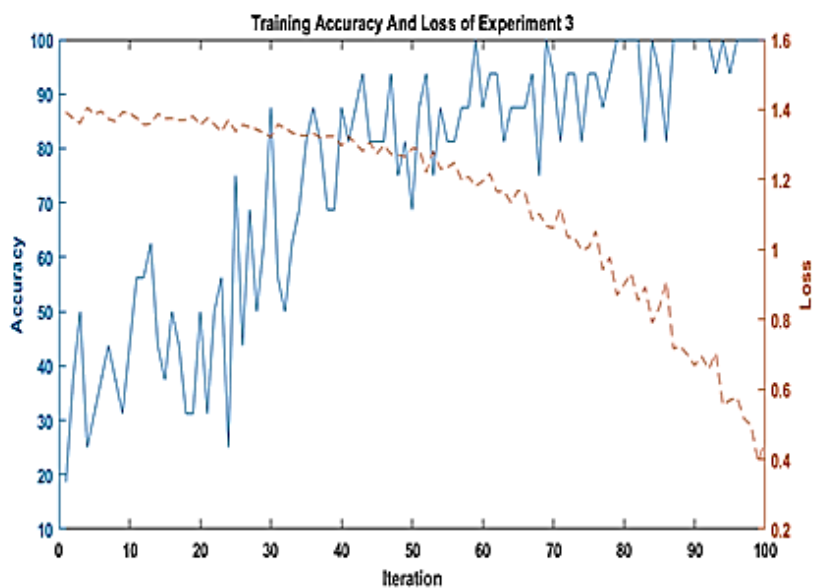

(b)

Figure 4. (a) ROC curve of experiment 3, (b) Training accuracy (\%) and loss of experiment 3

\subsection{Actual training data but erroneous test data}

Now, to confirm that our experiments are working perfectly, we replaced erroneous finger vein images to test data and check the validity of the experiments. To analyze furthermore the experimental data are given below. 


\subsubsection{Experiment 1}

In this experiment 1 , we trained the Alexnet as previously but replaced the four categories with several erroneous test data. This experiment 1 as shown in Figure 5 displays the training accuracy is $50 \%$ and AUC is 0.6. We know that $50 \%$ accuracy is not decent enough for recognition as well as the AUC is 0.6 which indicates a poor model and from ROC curve 5(a), we can state that it not a perfect model which is in fact true. In 5(b), training loss did not cross 1 but dropping the values which specifies that the model is working according but not with proper data.

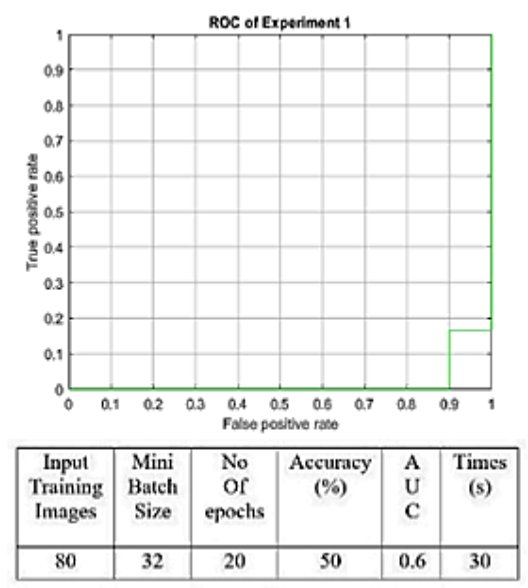

(a)

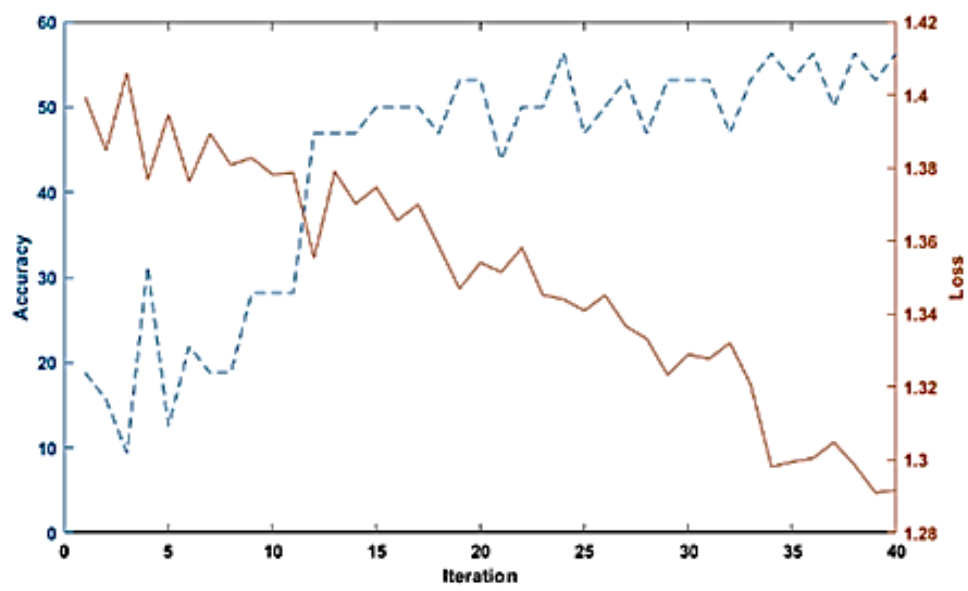

(b)

Figure 5. (a) ROC curve of Experiment 1, (b) Training Accuracy (\%) and loss of Experiment 1

\subsubsection{Experiment 2}

In the same way this experience is completed where in Figure 6(a) number of epochs and mini batch size is 32, but this time the training accuracy is zero percent and AUC is zero which directs that this model is not decent. Besides in Figure 6(b) the training loss goes downward and lands at 0 . Thus, we can say that our erroneous data has been testified that our model is working appropriately.

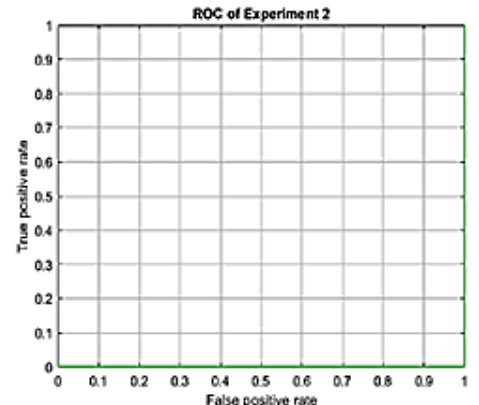

\begin{tabular}{|c|c|c|c|c|c|}
\hline $\begin{array}{c}\text { Input } \\
\text { Training } \\
\text { Images }\end{array}$ & $\begin{array}{c}\text { Mini } \\
\text { Batch } \\
\text { Size }\end{array}$ & $\begin{array}{c}\text { No } \\
\text { Of } \\
\text { epochs }\end{array}$ & $\begin{array}{c}\text { Accuracy } \\
\text { (\%) }\end{array}$ & $\begin{array}{c}\text { A } \\
\text { U } \\
\text { C }\end{array}$ & $\begin{array}{c}\text { Times } \\
(\mathrm{s})\end{array}$ \\
\hline 80 & 32 & 50 & 0 & 0 & 334 \\
\hline
\end{tabular}

(a)

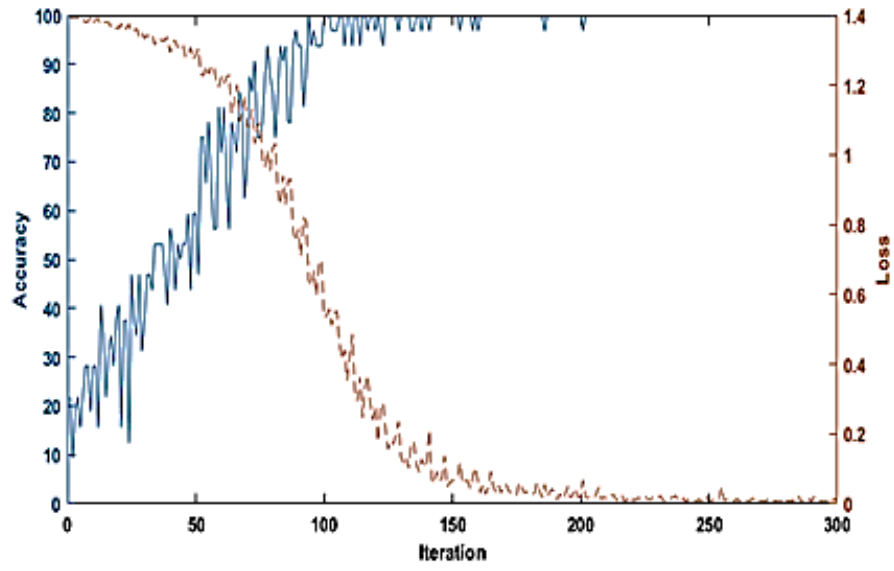

(b)

Figure 6. (a) ROC curve of experiment 2, (b) Training accuracy (\%) and loss of experiment 2 


\section{CONCLUSION}

In this paper, we introduced a transfer learning base CNN model which can recognize a finger vein with pre-trained test data. However, we analyzed and verified that the model is giving precise results by using both correct and erroneous test data. Further, we visualized the ROC curve and found experiment 3 in the previous section as a supreme model where used 20 epochs, 16 minibatch sizes and attained $100 \%$ training accuracy and $\mathrm{AUC}=1$ and in experiment 2 of section 3.2.2, was the worst example where accuracy $0 \%$ and $\mathrm{AUC}=0$ which means, this example predicted false classes. The outcome was satisfactory and, in the future, we would like to develop a real-time system based on these experimental results which will have the ability to identify finger veins in real time.

\section{ACKNOWLEDGEMENTS}

This work was conducted at IoT and Wireless Communication Protocols Laboratory, ECE Department, International Islamic University Malaysia (IIUM) and is partially funded by IIUM Publication RIGS grant no. P-RIGS19-003-0003.

\section{REFERENCES}

[1] J. F. Yang, Y. S. Shi, and J. L. Yang, "Person identification Based on finger-vein features," Computers in Human Behavior, vol. 28, pp. 1565- 1570, 2011

[2] J C. Hashimoto, "Finger vein authentication technology and its future," Symposium on VLSI Circuits Digest of Technical Papers, pp. 5-8, Honolulu, US, July 2006

[3] T. Yanagawa, S. Aoki, and T.Ohyama, "Human finger vein images are diverse and its patterns are useful for personal identification," Kyushu University.

[4] Yu Lu, Sook Yoon, Shan Juan Xi "Finger vein identification using polydirectional local line binary pattern", International Conference on ICT Convergence, 10.1109/ICTC.2013.6675307, 2013

[5] B. Besra and R. K. Mohapatra, "Extraction of segmented vein patterns using repeated line tracking algorithm," 2017 Third International Conference on Sensing, Signal Processing and Security (ICSSS),Chennai, 2017, pp. 89-92.

[6] G. C. Meng, A. Shahzad, N. M. Saad, A. S. Malik and F. Meriaudeau, "Prototype design for wearable veins localization system using near infrared imaging technique," 2015 IEEE 11th International Colloquium on Signal Processing \& Its Applications (CSPA), Kuala Lumpur, 2015, pp. 112-115.

[7] Ficke, B. W., Ransom, E. F., \& Oakes, J. E., "Near-Infrared Vein Visualization in Index Finger Pollicization", Journal of Hand Surgery, 42(6), 481.e1-481.e2. 2016.

[8] K. I. Ahmed, M. H. Habaebi, M. R. Islam and N. A. B. Zainal, "Enhanced vision based vein detection system," 2017 IEEE 4th International Conference on Smart Instrumentation, Measurement and Application (ICSIMA), Putrajaya, 2017, pp. 1-6.

[9] H. Qin, L. Qin, L. Xue, X. He, C. Yu, and X. Liang, "Finger-vein verification based on multi-features fusion," Sensors, vol. 13, no. 11, pp. 15 048-15 067, 2013

[10] L. Yang, G. Yang, Y. Yin and X. Xi, "Finger Vein Recognition With Anatomy Structure Analysis," in IEEE Transactions on Circuits and Systems for Video Technology, vol. 28, no. 8, pp. 1892-1905, Aug. 2018.

[11] E. C. Lee, H. Jung, and D. Kim, "New finger biometric method using near infrared imaging," Sensors, vol. 11, no. 3, pp. 2319-2333, 2011.

[12] J.-D. Wu and C.-T. Liu, "Finger-vein pattern identification using principal component analysis and the neural network technique," Expert Systems with Applications, vol. 38, no. 5, pp. 5423-5427, 2011.

[13] A. Kumar and Y. Zhou, "Human Identification Using Finger Images," in IEEE Transactions on Image Processing, vol. 21, no. 4, pp. 2228-2244, April 2012.

[14] N. Miura, A. Nagasaka, and T. Miyatake, "Feature extraction of finger- vein patterns based on repeated line tracking and its application to personal identification," Machine Vision and Applications, vol. 15, no. 4, pp. 194-203, 2004.

[15] J. Yang, Y. Shi, and G. Jia, "Finger-vein image matching based on adaptive curve transformation," Patt. Rec., vol. 66, pp. 34-43, 2017.

[16] F. Liu, G. Yang, Y. Yin, and S. Wang, "Singular value decomposition-based minutiae matching method for finger vein recognition," Neuro- computing, vol. 145, pp. 75 - 89, 2014

[17] R. Das, E. Piciucco, E. Maiorana and P. Campisi, "Convolutional Neural Network for Finger-Vein-Based Biometric Identification," in IEEE Transactions on Information Forensics and Security, vol. 14, no. 2, pp. 360-373, Feb. 2019.

[18] Mathworks, "Introducing Dee Learning with https://fr.mathworks.com/content/dam/mathworks/tagteam/Objects/d/80879v00_Deep_Learning_ebook.pdf,2017

[19] Subha Fairuz, Mohamed Hadi Habaebi, "Convolutional Neural Network-based Finger Vein Recognition using Near Infrared Images", 7th International Conference on Computer and Communication Engineering (ICCCE), 2018.

[20] Anibal Pedraza1, Jaime Gallego1, Samuel Lopez1, "Glomerulus classification with Convolutional Neural Networks", Communications in Computer and Information Science. June 2017

[21] https://www.mathworks.com/solutions/deeplearning/convolutional-neural-network.html

[22] Raghavendra, R Busch, C Tirunagar,"The $1^{\text {st }}$ Competition on Counter Measures to Finger Vein Spoofing Attacks", 
[23] S. Fairuz, M. H. Habaebi and E. M. A. Elsheikh, "Finger Vein Identification Based On Transfer Learning of AlexNet," 2018 7th International Conference on Computer and Communication Engineering (ICCCE), Kuala Lumpur, 2018, pp. 465-469.

[24] K. S. Itqan, A. R. Syafeeza, F. G. Gong, N. Mustafa,"User Identification System based on finger-vein Patterns using convolutional neural network", ARPN Journal of Engineering and Applied Sciences, 2016

[25] W. Liu, W. Li, L. Sun, L. Zhang and P. Chen, "Finger vein recognition based on deep learning," 2017 12th IEEE Conference on Industrial Electronics and Applications (ICIEA), Siem Reap, 2017, pp. 205-210.

[26] Hyung Gil Hong, Min Beom Lee and Kang Ryoung Park. "Convolutional Neural Network-Based Finger-Vein Recognition Using NIR Image Sensors”. Sensors (Basel). 2017 Jun 17(6): 1297.

[27] M. D. Zeiler and R. Fergus, "Visualizing and understanding convolutional neural networks,"Proceedings of the European Conference on Computer Vision, 2014

[28] A. Krizhevsky, I. Sutskever, G. E. Hinton, "Imagenet classification with deep convolutional neural networks," Advances in Neural Information Processing Systems, vol.2, pp. 1097-1105, 2012.

[29] L. Xiao, Q. Yan and S. Deng, "Scene classification with improved AlexNet model," 2017 12th International Conference on Intelligent Systems and Knowledge Engineering (ISKE), Nanjing, 2017, pp. 1-6.

[30] David J. Hand, "Measuring classifier performance: a coherent alternative to the area under the ROC curve", Mach Learn. 2009, 77: 103-123

[31] Teddy Surya Gunawan, Ahmad Fakhrur Razi Mohd Noor, Mira Kartiwi, "Development of English Handwritten Recognition Using Deep Neural Network," Indonesian Journal of Electrical Engineering and Computer Science, 10(2), pp. 562-568, 2018

\section{BIOGRAPHIES OF AUTHORS}

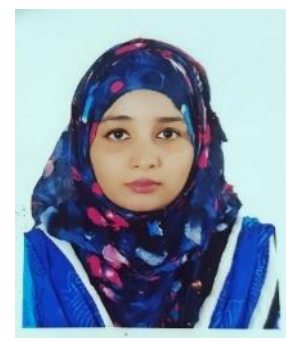

Subha Fairuz is pursuing her MSc in Computer and Information Engineering, International Islamic University Malaysia. Her research interests are in image processing, deep learning and IOT.

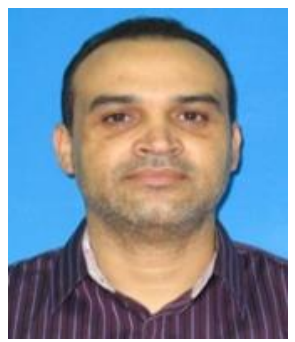

Mohamed Hadi Habaebi is with the department of electrical and Computer Engineering, International Islamic University Malaysia. His research interests are in IoT, wireless communications and Networking.

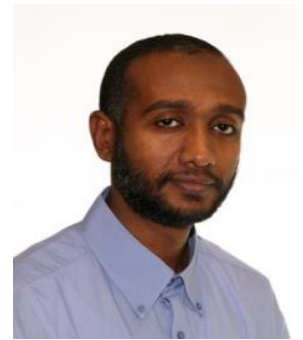

Dr Elsheikh Mohamed Ahmed Elsheikh currently works as an assisting professor at University Kuala Lumpur British Malaysian Institute in Selangor, Malaysia. He obtained his Ph.D. (Electronic and Electrical Engineering) and M.Sc. (Radio Systems Engineering) from University College London and University of Hull, respectively; in the United Kingdom. He obtained B.Sc. (Electrical and Electronic Engineering) from University of Khartoum in Sudan. Dr Elsheikh is interested in communication theory and information theory and their application to wireless communication. Current research include resource allocation and optimum operation techniques applied to cognitive radio networks and relay channels. 